\title{
On the discrepancies of graphs
}

\author{
József Balogh* \\ Department of Mathematics \\ University of Illinois at Urbana-Champaign \\ Illinois, U.S.A. \\ and Moscow Institute of Physics and Technology \\ Russian Federation \\ jobal@illinois.edu \\ Béla Csaba ${ }^{\dagger}$ \\ Bolyai Institute, Interdisciplinary Excellence Centre \\ University of Szeged \\ Szeged, Hungary \\ bcsaba@math.u-szeged.hu \\ Yifan Jing \\ András Pluhár ${ }^{\ddagger}$ \\ Department of Mathematics \\ University of Illinois at Urbana-Champaign \\ Illinois, U.S.A. \\ Department of Computer Science \\ University of Szeged \\ Szeged, Hungary \\ yifanjing17@gmail.com \\ pluhar@inf.u-szeged.hu
}

Submitted: Jan 4, 2019; Accepted: Mar 31, 2020; Published: Apr 17, 2020

(C) The authors. Released under the CC BY-ND license (International 4.0).

\begin{abstract}
In the literature, the notion of discrepancy is used in several contexts, even in the theory of graphs. Here, for a graph $G$ with each edge labelled -1 or 1 , we consider a family $\mathcal{S}_{G}$ of subgraphs of a certain type, such as spanning trees or Hamiltonian cycles. As usual, we seek for bounds on the sum of the labels that hold for all elements of $\mathcal{S}_{G}$, for every labeling.
\end{abstract}

Mathematics Subject Classifications: 05C35, 05D10, 11K38

*The first author is partially supported by NSF Grant DMS-1500121 and DMS-1764123, Arnold O. Beckman Research Award (UIUC Campus Research Board RB 18132) and the Langan Scholar Fund (UIUC).

${ }^{\dagger}$ The second author was partially supported by the Ministry of Human Capacities, Hungary, Grant 20391- 3/2018/FEKUSTRAT.

$\ddagger$ The second and fourth authors were partially supported by the NKFIH grants KH_18 129597 and SNN 117879. 


\section{Introduction}

The thorough study of discrepancy theory started with Weyl [14] and quickly gained several applications in number theory, combinatorics, ergodic theory, discrete geometry, statistics etc, see the monograph of Beck and Chen [3] or the book chapter by Alexander and Beck [1].

We touch upon only the combinatorial discrepancy of hypergraphs. Given a hypergraph $(X, E)$, and a mapping $f: X \rightarrow\{-1,1\}$, for an edge $A \in E$ let $f(A):=\sum_{x \in A} f(x)$. The discrepancy of $f$ is $\mathcal{D}(X, E, f)=\max _{A \in E}|f(A)|$, while the discrepancy of the hypergraph $(X, E)$

$$
\mathcal{D}(X, E):=\min _{f} \mathcal{D}(X, E, f) .
$$

In our case $X=E(G)$ and $E=\mathcal{S}_{G} \subset 2^{E(G)}$, and with a slight abuse of notation we write $\mathcal{D}\left(G, \mathcal{S}_{G}\right)$ for short.

Erdős, Füredi, Loebl, and Sós [11] studied the case $G=K_{n}$, the complete graph on $n$ vertices, and $\mathcal{S}_{G}$ is the set of copies of a fixed spanning tree $T_{n}$ with maximum degree $\Delta$. They showed the existence of a constant $c>0$, such that $\mathcal{D}\left(G, \mathcal{S}_{G}\right)>c(n-1-\Delta)$.

Erdös and Goldberg [10] defined $\operatorname{dis}(A, B):=e(A, B)-e(G)|A||B| /\left(\begin{array}{l}n \\ 2\end{array}\right)$, where $A, B \subset$ $V(G)$ and $A \cap B=\varnothing$. They showed that for every $\varepsilon>0$ there exists an $\varepsilon^{\prime}>0$ such that in every graph $G$ with $e=e(G)>v(G)=n$, there are disjoint sets $A, B \subset V(G)$, $|A|,|B| \leqslant \varepsilon n$, and $\operatorname{dis}(A, B)>\varepsilon^{\prime} \sqrt{e n}$.

Here we investigate the discrepancy of (spanning) trees, paths and Hamilton cycles. That is for a graph $G$ let $\mathcal{S}_{G}$ be the set of spanning trees $\left(\mathcal{T}_{n}\right)$, trees $(\mathcal{T})$, Hamiltonian paths $\left(\mathcal{P}_{n}\right)$, paths $(\mathcal{P})$, or Hamilton cycles $(\mathcal{H})$.

Usually, one expects big discrepancy if the hypergraph has many edges. Since for every graph $G$, either $G$ or $\bar{G}$ is connected, we have $\mathcal{D}\left(K_{n}, \mathcal{T}_{n}\right)=n-1$. Beck [2] showed that there is a graph $F$ on $n$ vertices and $2 n$ edges such that in every two-coloring of its edge set there exists a monochromatic path of length $c n$, that is $\mathcal{D}(F, \mathcal{P})=c n$. Another example for this is the interpretation of the result of Burr, Erdös and Spencer [6], namely that $R\left(m K_{3}, m K_{3}\right)=5 m$. That is if $k \cdot K_{3}$ is the set of triangle factors in $K_{n}, n=3 k$ and $n$ is divisible by 5 , then $\mathcal{D}\left(K_{n}, k \cdot K_{3}\right)=n / 5$.

We first consider the discrepancy of Hamilton cycles, and show that, roughly speaking, if $G$ has sufficiently large minimum degree then for every labeling of $E(G)$ with $+1,-1$ there is a Hamilton cycle with linear discrepancy.

Theorem 1. Let $c>0$ be an arbitrarily small constant and $n$ be sufficiently large. Let $G$ be a graph of order $n$ with $\delta(G) \geqslant(3 / 4+c) n$. Then we have $\mathcal{D}(G, \mathcal{H}) \geqslant c n / 32$.

Figure 1 below shows that the minimum degree condition in Theorem 1 is the best possible. In this example, let $G=K_{n}-K_{n / 4}$, i.e., $|V(G)|=n$ is divisible by $4,\left|V_{1}\right|=$ $n / 4,\left|V_{2}\right|=3 n / 4, \delta(G)=3 n / 4$. Assign -1 to all edges incident to $V_{1}$ and +1 to the rest of the edges. As each Hamilton cycle in $G$ touches $V_{1}$ exactly $n / 4$ times, they all have zero discrepancy. 


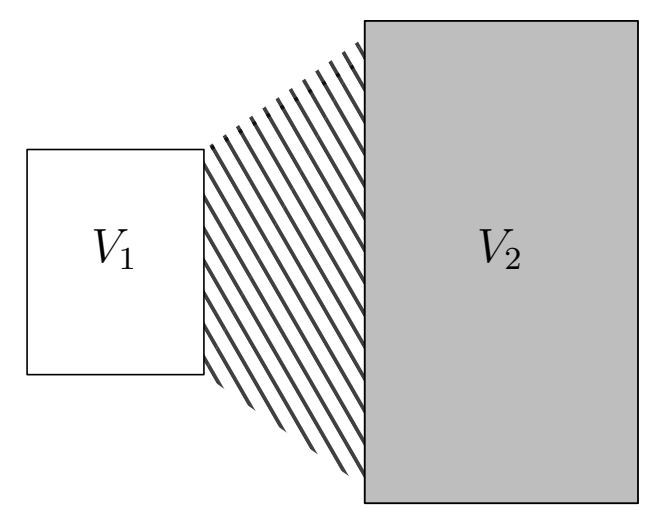

Figure 1: $G$ with $\delta(G)=3 n / 4$ and zero Hamilton cycle discrepancy.

For the existence of a Hamilton cycle, Dirac's Theorem requires only minimum degree $n / 2$. We could also push down the minimum degree requirement for the existence of a linear discrepancy Hamilton cycle, if we have some local restriction on the coloring.

For $\nu>0$ real number, we say a vertex is $\nu$-balanced if it has at least $\nu n$ edges with label +1 , and at least $\nu n$ edges of label -1 , otherwise it is $\nu$-unbalanced.

Theorem 2. Let $c, d, \nu$ be positive numbers satisfying $c \geqslant 8 \nu$ and $d \geqslant 4 \nu$. Let $G$ be a graph of order $n$, where $\delta(G) \geqslant(1 / 2+c) n$. Assume that the edges of $G$ are labelled by either +1 or -1 , such that the number of $\nu$-balanced vertices is at least $(3 / 4+d) n$. Then there exists a Hamilton cycle in $G$ with discrepancy at least $\nu^{2} n / 2000$.

The number of the balanced vertices in the graph in Figure 1 is $3 n / 4$, hence the condition on the size of the balanced set in Theorem 2 is tight.

In both of the theorems above, $G$ is dense. However, the sparsity of a graph does not imply small discrepancy, the expansion is a more important factor. Let $G \in \mathcal{G}_{n, d}$ be a randomly, uniformly selected $d$-regular graph on $n$ vertices. A property $\mathcal{P}$ holds with high probability, w.h.p., if for every $\varepsilon>0$ there exist an $n_{\varepsilon}$ such that $\operatorname{Pr}(G \in$ $\left.\mathcal{G}_{n, d}, G \in \mathcal{P}\right) \geqslant 1-\varepsilon$. Similarly, property $\mathcal{P}$ holds asymptotically almost surely, a.a.s., if $\lim _{n \rightarrow \infty} \operatorname{Pr}\left(G \in \mathcal{G}_{n, d}, G \in \mathcal{P}\right)=1$.

Theorem 3. Let $G \in \mathcal{G}_{n, 3}$. Then there exists a constant $c>0$ such that a.a.s. we have $\mathcal{D}\left(G, \mathcal{T}_{n}\right) \geqslant c n$.

For planar graphs, one can expect sublinear discrepancy of spanning trees; we managed to give asymptotically sharp bounds.

Theorem 4. Let $G$ be a planar graph on $n$ vertices. Then there exists a real number $c>0$ such that $\mathcal{D}\left(G, \mathcal{T}_{n}\right) \leqslant c \sqrt{n}$.

The bounds, up to the constant factor are best possible. Let $P_{k}^{2}:=P_{k} \square P_{k}$ be the $k \times k$ grid.

Theorem 5. $\mathcal{D}\left(P_{k}^{2}, \mathcal{T}_{n}\right) \geqslant c k$ for some $c>0$, where $n=k^{2}$. 
If we drop the condition of spanning subgraph, then the discrepancies can be linear in the number of vertices.

Proposition 6. Let $k, \ell$ be some positive integers. Then

$$
\mathcal{D}\left(P_{k} \square P_{\ell}, \mathcal{P}\right)>k \ell / 8-\max \{k, \ell\} / 8-\min \{k, \ell\} .
$$

We have the following corollary since paths are also trees.

Corollary 7. $\mathcal{D}\left(P_{k} \square P_{\ell}, \mathcal{T}\right)>k \ell / 8-\max \{k, \ell\} / 8-\min \{k, \ell\}$.

Let us make some easy observations which nevertheless give motivations for the above theorems and to those proofs. The graph $P_{2} \square P_{k}$ has exponentially many spanning trees, but still $\mathcal{D}\left(P_{2} \square P_{k}, \mathcal{T}_{2 k}\right) \leqslant 3$. To see this, we partition the graph into a $2 \times\lceil k / 2\rceil$ grid and a $2 \times\lfloor k / 2\rfloor$ grid, and label the edges of the first grid by -1 , of the second grid by +1 . We label the edge shared by two sub-grids arbitrarily. The situation for $P_{k} \square P_{k}$, the $k \times k$ grid, is similar: cut the grid into two halves and label +1 the upper, and -1 the lower region. Since any spanning tree is cut at most $k$ times, $\mathcal{D}\left(P_{k} \square P_{k}, \mathcal{T}_{n}\right) \leqslant k-1$. For not necessarily spanning trees, obviously, $\mathcal{D}(G, \mathcal{T}) \geqslant\lceil\Delta(G) / 2\rceil$.

\section{Notation}

We let $N^{+}(v)$ to denote the set of neighbors $u$ of $v$ such that $u v$ is labelled by +1 . Similarly, $N^{-}(v)$ denotes the set of neighbors $u$ of $v$ such that $u v$ is labelled by -1 . By definition $N(v)=N^{+}(v) \cup N^{-}(v)$. We let $d^{+}(v)=\left|N^{+}(v)\right|, d^{-}(v)=\left|N^{-}(v)\right|$ and $d(v)=$ $d^{+}(v)+d^{-}(v)$. Suppose $U \subseteq V(G)$, we define $N(U)=\{v \in V(G) \mid \exists u \in U, u v \in E(G)\}$. We say $u$ is a positive neighbor of $v$ if $u \in N^{+}(v)$, and $u$ is a negative neighbor of $v$ if $u \in N^{-}(v)$. Suppose $H$ is a subgraph of $G$, we define $f(H)$ to be the sum of labels of all the edges of $H$, where $f: E(G) \rightarrow\{1,-1\}$.

\section{Structure of the paper}

The paper is organized as follows. In Section 2, we discuss the discrepancy of Hamilton cycles. In Section 3, we prove Theorem 3 for random 3-regular graphs. Section 4 contains some results of discrepancies for grids and planar graphs.

\section{Discrepancy of Hamilton cycles}

In this section, we study the discrepancy of Hamilton cycles. The first tool we use is the following generalization of Dirac's Theorem [13].

Lemma 8. Let $G=(V, E)$ be a graph and let $c>0$ be a real number. Suppose $E^{\prime} \subseteq E$ such that $E^{\prime}$ induces a linear forest in $G$. If $\delta(G) \geqslant\left(\frac{1}{2}+c\right) n$ and $\left|E^{\prime}\right| \leqslant 2 c n$, then there exists a Hamilton cycle in $G$ which contains all the edges in $E^{\prime}$.

We will use the following simple lemma at various points: 
Lemma 9. Let $\nu, \gamma>0$. Suppose $U \subseteq V(G)$ with $|U| \geqslant \nu n$ such that for every $u \in U$, we have $|N(u)| \geqslant \gamma n$. Then there exists a path $P$ of length at least $\nu \gamma n / 2$, such that every edge in $P$ contains at least one vertex in $U$. Moreover, if for every $u \in U$ we have $|N(u) \backslash U| \geqslant \gamma n$, then there exists a path of length at least $\nu \gamma n$ whose vertices are alternating between $U$ and $N(U) \backslash U$.

Proof. Let $H$ be the collection of edges incident to the vertices in $U$. We have $e(H) \geqslant$ $\nu \gamma n^{2} / 2$. This implies $H$ contains a path $P$ of length at least $\nu \gamma n / 2$. It is clear that $P$ satisfies all the requirements. The second part of the statement follows very similarly, considering edges only having exactly one endpoint in $U$.

Let $G$ be an $n$-vertex simple graph with $\delta(G) \geqslant(3 / 4+c) n$, where $c>0$ is a (possibly small) constant and the edges of $G$ are labelled by either +1 or -1 .

Proof of Theorem 1. Let $a=c / 4$. The proof is split into two cases.

Case 1: At least $(3 / 4+c) n$ vertices in $G$ are a-balanced.

Suppose there exists a vertex $v$ such that less than $c n / 2$ vertices in $N(v)$ have more than $c n$ negative neighbors in $N(v)$. Let $M \subseteq N(v)$ be the set of such vertices, hence, $|M|<c n / 2$. Note that each vertex in $N(v)$ has at least $(1 / 2+2 c) n$ neighbors inside $N(v)$, hence $G[N(v) \backslash M]$ contains a Hamilton cycle $H$ with all edges being positive. Then we insert those vertices not in $N(v) \backslash M$ one by one to $H$, so we obtain a Hamilton cycle with discrepancy at least

$$
|N(v) \backslash M|-3|V \backslash(N(v) \backslash M)| \geqslant 2 c n .
$$

Now suppose that such vertex does not exist. Let $S \subseteq V(G)$ be the set of balanced vertices which have more positive neighbors. We may assume $|S| \geqslant(3 / 4+c) n / 2$. Then for every $v \in S$, each vertex in $N^{+}(v)$ has at least $(1 / 8+3 c / 2) n$ neighbors in $N^{+}(v)$. Every vertex of $S$ has at least a negative neighbors, hence, using Lemma 9 we get that there exists a negative path $P$, such that each edge of $P$ contains at least one vertex in $S$, and both of the end vertices of $P$ are in $S$. Moreover, the length of $P$ is at least $\frac{a n}{2}\left(\frac{3}{4}+c\right)$. We denote the end vertices of $P$ by $x, y$.

Next for each vertex $v$ in $V(P) \cap S$, we pick an edge in $N^{+}(v)$. For each vertex in $P$ but not in $S$, we pick a negative edge from its neighborhood. We also pick an edge $a b$ such that $a \sim x$ and $b \sim y$. We require that all the edges we picked are disjoint from $P$ and they form a linear forest in $G$. This is doable, since in each step we forbid edges incident to the vertices in $V^{\prime} \subseteq V(G)$ with $\left|V^{\prime}\right|<c n / 2$.

Let $G^{\prime}$ be the graph after we delete $P$ from $G$. By Lemma 8, there is a Hamilton cycle $H$ in $G^{\prime}$ containing all the edges we picked. First, we insert the entire path $P$ by removing the $a b$ edge and adding edges $a x$ and $b y$. We obtain a Hamilton cycle $H_{1}$, such that

$$
f\left(H_{1}\right) \leqslant f(H)-|P|+3 \leqslant f(H)-\frac{a n}{2}\left(\frac{3}{4}+c\right)+3 .
$$


If $f(H) \leqslant 3 c n / 64$, then the above implies that $f\left(H_{1}\right) \leqslant-3 c n / 64$. If $f(H)>3 c n / 64$, then we can insert the vertices in $P$ one by one to obtain $H_{2}$, such that we have

$$
f\left(H_{2}\right) \geqslant f(H)+\frac{|P|}{2}-\frac{|P|}{2}=f(H) .
$$

Therefore, $G$ contains a Hamilton cycle with discrepancy at least $3 \mathrm{cn} / 64$.

Case 2: There are at least $2 c n$ vertices in $G$ which are not a-balanced.

Suppose there exists a set $T$ containing $c n$ unbalanced vertices, each having at most an negative neighbors. Let $H$ be a Hamilton cycle in $G$. The difference between the number of positive edges and negative edges of $H$ is at most $c n$, otherwise we are done. Then for every vertex $v \in T, N^{+}(v)$ contains at least $\left(2 c-2 a-\frac{c}{2}\right) n$ positive edges of $H$ and at least $\left(2 c-2 a-\frac{c}{2}\right) n$ negative edges of $H$. For each vertex $v$ in $T$, we pick a positive edge and a negative edge in $N^{+}(v) \cap H$.

Now we define $G^{\prime}:=G-T$. By Lemma 8 , there is a Hamilton cycle $H$ in $G^{\prime}$ that contains all the edges we picked. We can either remove all the negative edges we picked in $H^{\prime}$ to insert the vertices in $T$, or remove all the positive edges we picked. Clearly, $G$ contains a Hamilton cycle with discrepancy at least $c n$.

Now we need some preparation to prove Theorem 2. Let $\mathfrak{T}$ be the set of triangles in $G$. We define a function

$$
g: V(G) \times \mathfrak{T} \rightarrow\{-3,-1,0,1,3\},
$$

such that for every $v \in V(G)$ and triangle $T \in \mathfrak{T}$, we let $g(v, T)=0$ if $v$ is not a vertex in $T$. For $T=u v w$, we let $g(v, T)$ be the change in the discrepancy if the edge $u w$ is changed to the path uvw. To be more precise, we let $g(v, T)$ be $-1,1,-3,3$ if the triangle $T$ has type red, blue, dark red, dark blue, respectively, see Figure 2.

We color the vertex $v$ red if there exist at least $\nu n^{2}$ triangles $T$ in $\mathfrak{T}$ such that $g(v, T)=$ -1 , it is blue, dark red, dark blue if there exist at least $\nu n^{2}$ triangles $T$ in $\mathfrak{T}$ such that $g(v, T)=1,-3,3$, respectively. Note that when $c>8 \nu$, every vertex is colored, since the neighborhood of every vertex spans at least $\mathrm{cn}^{2} / 2$ edges. Some vertices may have multiple colors under this definition, but we may assume most of them have only one color, using the following lemma.

Lemma 10. Suppose more than $\nu n / 3$ vertices have more than one colors. Then there exists a Hamilton cycle of discrepancy at least $\nu n / 3$.

Proof. Let $M \subseteq V(G)$ be the set of vertices having more than one colors, and $x_{1}, x_{2}, \ldots$, $x_{\nu n / 3} \in M$. For every $x_{i}(1 \leqslant i \leqslant \nu n / 3)$, we pick edges $a_{i} b_{i}$ and $c_{i} d_{i}$ in the neighborhood of $x_{i}$, such that $g\left(x_{i}, x_{i} a_{i} b_{i}\right) \neq g\left(x_{i}, x_{i} c_{i} d_{i}\right)$. We further require that, all the edges we picked do not contain $x_{i}$, and they form a linear forest in $G$. We can do this, since in each step we forbid less than $\nu n^{2}$ edges, but we have at least $\nu n^{2}$ triangles by the definition.

Now we remove $x_{1}, x_{2}, \ldots, x_{\nu n / 3}$ from $G$, and call the resulted graph $G^{\prime}$. By Lemma 8 , we can find a Hamilton cycle $H$ in $G^{\prime}$ containing all the edges we picked. In order to insert 
$x_{i}$ back to $H$, we can remove either $a_{i} b_{i}$ or $c_{i} d_{i}$, and in each step, the discrepancies differ by at least 2 , since $\left|g\left(x_{i}, x_{i} a_{i} b_{i}\right)-g\left(x_{i}, x_{i} c_{i} d_{i}\right)\right| \geqslant 2$. Therefore, there exists a Hamilton cycle in $G$ with discrepancy at least $\nu n / 3$.

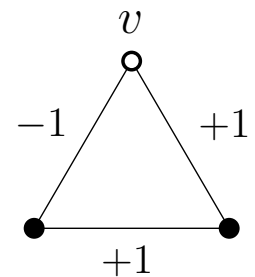

$+1$

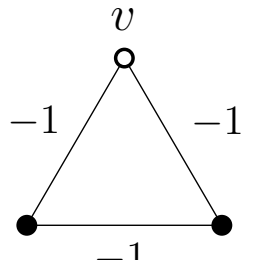

$-1$

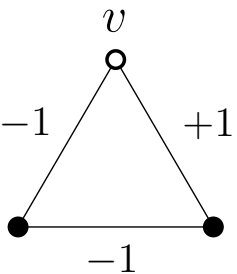

Type blue.

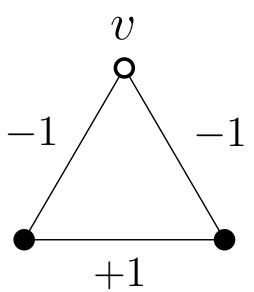

Type dark red.

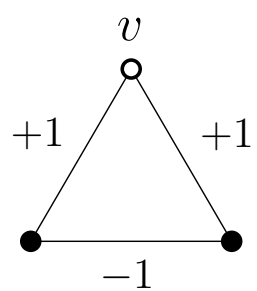

Type dark blue.

Figure 2: The vertex coloring of $G$.

The following Lemma is our main tool in the proof.

Lemma 11. Let $c, \nu>0$ with $c>8 \nu$. Let $G$ be a graph with $\delta(G) \geqslant(1 / 2+c) n$. Let $R, Q \subseteq\{$ red, blue, dark red, dark blue $\}$. Suppose, there is a path $P$ of length $\phi(\nu) n$ for some function $\phi$ and all edges of it have labels in $I \subseteq\{+1,-1\}$, where $0<\phi(\nu)<\nu / 2$, and each edge of $P$ contains at least one vertex with colors in $R$, and the other vertices on $P$ have colors in $Q$. Assume that one of the following holds:

(i) $I=\{-1\}, R=\{$ dark blue $\}$.

(ii) $I=\{-1\}, R=\{$ blue $\}$, dark red $\notin Q$.

(iii) $R=Q=\{$ dark blue $\}$.

(iv) $R=\{$ dark blue $\}, Q=\{$ blue $\}$.

(v) $I=\{+1\}, R=Q=\{$ red $\}$.

Then $G$ contains a Hamilton cycle with discrepancy at least $\phi(\nu) n / 2-3 / 2$.

Proof. Let $X$ be the set of vertices on $P$ with colors in $R$, and let $Y$ be the set of vertices with colors in $Q$. Suppose $x, y$ are the first and the last vertices in $P$.

Let us focus on (i) first. For every vertex $v \in X$, we pick an edge $a_{v} b_{v}$ inside the neighborhood of $v$, such that $a_{v}, b_{v} \notin V(P)$, and $g\left(v, v a_{v} b_{v}\right)=3$. We require that the edges we picked form a linear forest. This is possible, and we can pick the edges one by one. For each step, the edge we chose cannot contain a vertex which already used twice 
in the previously chosen edges, and two end vertices of the new edge cannot both already used. Clearly, the number of edges that cannot be chosen is strictly less than $\nu n^{2}$, but we have $\nu n^{2}$ options, by the definition of the dark blue vertices.

For every vertex $u$ in $Y$, we pick an edge $a_{u} b_{u}$ in $N(u)$, and we pick the edge $a b$ such that $a \sim x$ and $b \sim y$, so for the endpoints $x$ and $y$ we pick two edges. Together with the edges we picked for the vertices in $X$, we further require that all the edges we picked are disjoint from $P$ and they form a linear forest in $G$. Note that the number of edges we picked is less than $c n$.

Let $G^{\prime}$ be the graph after removing all the vertices in $P$ from $G$, we have $\delta\left(G^{\prime}\right) \geqslant$ $(1 / 2+c / 2) n$. Now we apply Lemma 8 , and suppose $H$ is a Hamilton cycle in $G^{\prime}$ containing all the edges we picked. We have two different ways to construct a Hamilton cycle in $G$.

We remove the edge $a b$ and add $a x, b y$ to insert the entire path $P$, we denote the resulted Hamilton cycle by $H_{1}$. Clearly,

$$
f\left(H_{1}\right) \leqslant f(H)-|P|+3=f(H)-\phi(\nu) n+3 .
$$

We can also insert the vertices in $P$ one by one. That is, for every $v \in V(P)$, we remove the edge $a_{v} b_{v}$ in $H$ and add the edges $v a_{v}, v b_{v}$. We then obtain a Hamilton cycle $\mathrm{H}_{2}$, and we have

$$
f\left(H_{2}\right) \geqslant f(H)+3|X|-3|Y|=f(H),
$$

since the worst case is when all the vertices in $Y$ are dark red. Therefore, we obtain a Hamilton cycle in $G$ with discrepancy at least $\frac{1}{2}(\phi(\nu) n-3)$.

Now we consider (ii). The ideas are similar: For every vertex $v$ in $X$, we pick an edge $a_{v} b_{v}$ in $N(v)$ such that the $g\left(v, v a_{v} b_{v}\right)=1$. For every vertex $u$ in $Y$, we pick an edge $a_{u} b_{u}$ in $N(u)$ such that $g\left(u, u a_{u} b_{u}\right) \neq-3$. We also pick $a b$ adjacent to the end vertices of $P$, and we require all the edges we picked are disjoint from $P$, and they form a linear forest.

We now remove all the vertices in $P$ from $G$. Let $H$ be the Hamilton cycle in the resulted graph which contains all the edges we picked. We can either insert the entire path to $H$, or insert the vertices one by one. In the second situation, the worst case is when all the vertices in $Y$ are red. This gives us a Hamilton cycle with discrepancy at least $\frac{1}{2}(\phi(\nu) n-3)$.

Note that (ii) implies (v), since we can map -1 to +1 , blue to red, and dark blue to dark red. For cases (iii) and (iv), for vertices in $X$, we pick edges as we did in (i). For the vertex $u$ in $Y$, we pick $a_{u} b_{u}$ in $N(u)$ such that $g\left(u, u a_{u} b_{u}\right)$ is 3 and 1 , respectively. Again, we construct a new graph $G^{\prime}$ by removing vertices in the path $P$, and we find a Hamilton cycle $H_{1}$ in $G^{\prime}$ containing all the edges we picked. We still have two ways to obtain the Hamilton cycle in $G$, insert the entire path, or insert the vertices one by one. If we insert the entire path, denote the resulted Hamilton cycle by $H_{1}$, we get

$$
f\left(H_{1}\right) \leqslant f(H)+|P|+3=f(H)+\phi(\nu) n+3 .
$$

If we insert the vertices in $P$ one by one, denote the resulted Hamilton cycle by $\mathrm{H}_{2}$, we have

$$
f\left(H_{2}\right) \geqslant f(H)+3|X|+|Y| \geqslant f(H)+2 \phi(\nu) n .
$$

Thus we obtain a Hamilton cycle in $G$ with discrepancy at least $\frac{1}{2}(\phi(\nu) n-3)$. 
Remark. Note that if we reverse the colors and the labels simultaneously, the same conclusions in Lemma 11 still hold.

With all tools in hand, we are going to prove Theorem 2.

Proof of Theorem 2. Let $M \subseteq V(G)$ be the set of vertices having more than 1 colors. By Lemma 10, we have $|M|<\nu n / 3$. Let $A, B, C, D \subseteq V(G) \backslash M$ be the set of blue, red, dark blue and dark red vertices, respectively. By Lemmas 9 and 11, we may assume the following properties of $G$.

(i) At most $\nu n / 30$ vertices in $C(D)$ have more than $\nu n / 4$ neighbors in $C(D)$. Otherwise by Lemma 9 we can find a path $P$ of length $\nu^{2} n / 240$ either inside $C$, or inside $D$. In both cases, condition (iii) in Lemma 11 gives us a Hamilton cycle of discrepancy at least $\nu^{2} n / 480-3 / 4$.

(ii) At most $\nu n / 30$ vertices in $C(D)$ have more than $\nu n / 4$ neighbors in $A(B)$. If not, there is a path of length $\nu^{2} n / 120$ whose vertices alternate between $C$ and $A$ (between $D$ and $B$ ), and condition (iv) in Lemma 11 gives us a Hamilton cycle of discrepancy at least $\nu^{2} n / 240-3 / 2$.

(iii) At most $\nu n / 3$ vertices in $A(B)$ have more than $\nu n / 6$ negative (positive) neighbors inside $A(B)$. By the same reason as above, otherwise condition (v) in Lemma 11 gives us a Hamilton cycle of discrepancy at least $\nu^{2} n / 72-3 / 2$.

(iv) At most $\nu n / 30$ vertices in $C(D)$ have more than $\nu n / 4$ neighbors in $D(C)$. If, say, at least $\nu n / 30$ vertices in $C$ have more than $\nu n / 4$ neighbors in $D$, then suppose $\nu n / 60$ of them have more positive neighbors in $D$. By Lemma 9 , there is a positive path $P$ of length $\nu^{2} n / 960$ whose vertices alternate between $C$ and $D$. We now apply condition (i) in Lemma 11, but in the form that $I=\{+1\}$ and $R=\{$ dark red $\}$. Thus there exists a Hamilton cycle with discrepancy at least $\nu^{2} n / 1920-3 / 2$.

(v) At most $\nu n / 3$ vertices in $A(B)$ have more than $\nu n / 6$ neighbors in $B(A)$. By the same reason as above, if we have more than $\nu n / 3$ vertices in $A$ having more than $\nu n / 6$ neighbors in $B$, we may suppose that $\nu n / 6$ of them have more positive neighbors in $B$. Thus by Lemma 9 there is a path of length $\nu^{2} n / 144$ whose vertices alternate between $A$ and $B$. Then we apply condition (ii) in Lemma 11 with $I=\{+1\}$ and $R=\{$ red $\}$, there exists a Hamilton cycle with discrepancy at least $\nu^{2} n / 288-3 / 2$.

(vi) At most $\nu n / 30$ vertices in $C(D)$ have more than $\nu n / 4$ negative (positive) neighbors in $B(A)$. Otherwise condition (i) in Lemma 11 gives a Hamilton cycle of discrepancy at least $\nu^{2} n / 240-3 / 2$.

(vii) At most $\nu n / 3$ vertices in $A(B)$ have more than $\nu n / 6$ neighbors in $C(D)$. If not, the condition (iv) in Lemma 11 gives a Hamilton cycle of discrepancy at least $\nu^{2} n / 72-3 / 2$.

(viii) At most $\nu n / 3$ vertices in $A(B)$ have more than $\nu n / 6$ positive (negative) neighbors in $D(C)$. If not, the condition (i) in Lemma 11 gives a Hamilton cycle of discrepancy at least $\nu^{2} n / 36-3 / 2$.

Now the approximate structure of $G$ is as follows. The graph induced on $C \cup D$ is almost empty, and $G[A, C], G[B, D], G[A, B]$ are almost empty. Almost all the edges 
between $A$ and $D$ are negative, and almost all the edges between $B$ and $C$ are positive. Almost all the edges inside $A$ are positive, and almost all the edges inside $B$ are negative.

We say a vertex in a set is typical if it behaves as almost all the vertices in this set, otherwise it is untypical. More precisely, a vertex $v \in A(B)$ is typical if it has less than $\nu n / 6$ negative (positive) neighbors in $A(B)$, less than $\nu n / 6$ neighbors in $B(A)$, less than $\nu n / 6$ neighbors in $C(D)$, and less than $\nu n / 6$ positive neighbors in $D(C)$. A vertex $v \in C$ $(D)$ is typical if it has less than $\nu n / 4$ neighbors in $C(D)$, less than $\nu n / 4$ neighbors in $A$ $(B)$, less than $\nu n / 4$ neighbors in $D(C)$, and less than $\nu n / 4$ negative (positive) neighbors in $B(A)$.

The rest of the proof is based on analyzing the number of dark vertices.

Case 1: There exist at most $\nu n / 6$ dark blue vertices and at most $\nu n / 6$ dark red vertices.

In this case, we have $|A \cup B| \geqslant\left(1-\frac{\nu}{3}\right) n$. Suppose $|A| \geqslant\left(\frac{1}{2}-\frac{\nu}{6}\right) n$, and let $A^{\prime} \subseteq A$ be the set of $\nu$-balanced vertices. Clearly, $\left|A^{\prime}\right| \geqslant\left(\frac{1}{4}+d-\frac{\nu}{6}\right) n$, and each vertex $v \in A^{\prime}$ has at least $\nu n$ negative neighbors. By (iii), (v) and (vii), all but $\nu n$ vertices in $A^{\prime}$ have less than $\nu n / 6$ negative neighbors inside $A$, in $B$ and in $C$. Since $|D| \leqslant \nu n / 6$ and $|M|<\nu n / 3$, we get a contradiction.

Case 2: There are at least $\nu n / 6$ dark blue vertices or $\nu n / 6$ dark red vertices.

Suppose $|C| \geqslant \nu n / 6$. By (i), (ii), (iv), and (vi), we have that at most $2 \nu n / 15$ vertices in $C$ are untypical, which implies that all the other vertices in $C$ are $\nu$-unbalanced. Hence $|C| \leqslant\left(\frac{1}{4}-d+\frac{2 \nu}{15}\right) n$, and $|B| \geqslant\left(\frac{1}{2}+c-\frac{3}{4} \nu\right) n$, since the typical vertices in $C$ have at most $3 \nu / 4$ vertices outside of $B$. This also gives us $|D| \leqslant \nu n / 6$, otherwise we would also have $|A| \geqslant\left(\frac{1}{2}+c-\frac{3 \nu}{4}\right) n$, and this contradicts with $A \cap B=\varnothing$.

Thus, we have $|A| \leqslant\left(\frac{1}{2}-c+\frac{7 \nu}{12}\right) n$, and actually this implies $|A| \leqslant 4 \nu n / 3$. The reason for this is, first by (iii), (v), (vii), and (viii), $A$ contains at most $4 \nu n / 3$ untypical vertices. By (v) and (vii), the typical vertices in $A$ have all but at most $\nu n / 3$ of their neighbors in $A$ and $D$. But $|A \cup D| \leqslant\left(\frac{1}{2}-c+\frac{3 \nu}{4}\right) n$, which means that all the vertices in $A$ are untypical because of the disjointness of $A, B, C, D, M$. Therefore, we have $|A \cup C \cup D \cup M| \leqslant\left(\frac{1}{4}-d+2 \nu\right) n$, and thus $|B| \geqslant\left(\frac{3}{4}+d-2 \nu\right) n$.

Let $B^{\prime} \subseteq B$ be the set of typical vertices in $B$, and let $C^{\prime} \subseteq C$ be the set of typical vertices in $C$. Clearly, we have $\left|B \backslash B^{\prime}\right| \leqslant \nu n$ and $\left|C \backslash C^{\prime}\right| \leqslant \frac{2}{15} \nu n$. Let $K$ be a graph such that $V(K)=B^{\prime} \cup C^{\prime}$, and $e \in E(K)$ if $e$ is either a negative edge in $G\left[B^{\prime}\right]$ or a positive edge in $G\left[B^{\prime}, C^{\prime}\right]$. Now, $|V(K)| \geqslant(1-3 \nu) n$, since besides $B \backslash B^{\prime}$ and $C \backslash C^{\prime}$, we have $|A| \leqslant 4 \nu n / 3,|D| \leqslant \nu n / 6$, and $|M| \leqslant \nu n / 3$. Also, for every $v \in V(K), \delta_{K}(v) \geqslant$ $\left(\frac{1}{2}+c-3 \nu\right) n$. This is because, for every $u \in B^{\prime}$, by (v) and the size of $D$ and $M$, all but at most $2 \nu n / 3$ neighbors of $u$ are in $B \cup C$. By (iii) and (viii), $u$ has at most $\nu n / 6$ positive neighbors in $B$ and at most $\nu n / 6$ negative neighbors in $C$. By the size of $B \backslash B^{\prime}$ and $C \backslash C^{\prime}$, we have $\delta_{K}(u) \geqslant\left(\frac{1}{2}+c\right) n-2 \nu n-\frac{2 \nu n}{15}$. Similarly, for every $w \in C^{\prime}$, by (i), (ii), (iv), (vi), and the size of $M$ and $B \backslash B^{\prime}, \delta_{K}(w) \leqslant\left(\frac{1}{2}+c\right) n-2 \nu n-\frac{\nu n}{3}$. Therefore, $K$ contains a Hamilton cycle $H$. Since $C^{\prime}$ is an independent set in $K$ by (i), the number of positive edges in $H$ is at most $2\left|C^{\prime}\right| \leqslant 2|C| \leqslant\left(\frac{1}{2}-2 d+\nu\right) n$.

Now we go back to $G$. Note that $H$ is also a Hamilton cycle in $G\left[B^{\prime} \cup C^{\prime}\right]$. In the final step, we are going to insert all the vertices in $V(G) \backslash\left(B^{\prime} \cup C^{\prime}\right)$ to $H$. Let $J=\left(B \backslash B^{\prime}\right) \cup\left(C \backslash C^{\prime}\right) \cup A \cup D \cup M$. We have $|J| \leqslant 3 \nu n$. Then after we insert all vertices 
in $J$ to $H$, we obtain a Hamilton cycle in $G$, which contains at most $\left(\frac{1}{2}-2 d+\nu\right) n+2|J|=$ $\left(\frac{1}{2}-2 d+7 \nu\right) n$ positive edges. Therefore, $G$ contains a Hamilton cycle with discrepancy at least $(4 d-14 \nu) n>2 \nu n$.

\section{Discrepancies in random 3-regular graphs}

Proof of Theorem 3. Buser [7] and later, in a much simpler paper, Bollobás [5] showed that random regular graphs have expanding properties. More precisely, let

$$
i(G):=\min _{U} \frac{|\partial U|}{|U|}
$$

where $U \subset V(G)$ with $|U| \leqslant|V(G)| / 2$, and $\partial U:=\{v \notin U \mid \exists u \in U, u v \in E(G)\}$.

(i) Bollobás [5] proved that $i(G) \geqslant 2^{-7}$ for a random 3-regular graph $G$ with high probability. In particular, it is connected w.h.p..

(ii) Bollobás [4] showed for $3 \leqslant j \leqslant k$, where $k$ is fixed, and $X_{j}$ stands for the number of cycles of length $j$ in $G \in \mathcal{G}_{n, 3}$, that $X_{3}, \ldots, X_{k}$ are asymptotically independent Poisson random variables with means $\lambda_{j}=2^{j} /(2 j)$.

(iii) Wormald proved (see [15, Lemma 2.7]) that for a fixed $d$ and every fixed graph $F$ with more edges than vertices, $G \in \mathcal{G}_{n, d}$ a.a.s. contains no subgraph isomorphic to $F$.

Fix an arbitrary $f: E(G) \rightarrow\{-1,1\}$, denote $N$ and $P$ the subsets of edges, where $f$ takes -1 and 1 , respectively. We may assume that $|N| \leqslant|P|$, i.e., $|N| \leqslant 3 n / 4$.

Denote by $G^{+}$the subgraph of $G$ spanned by $P$, and let $A_{i}$ be the set of components with size $i$ in $G^{+}$, while $a_{i}:=\left|A_{i}\right|$. The number of components in $G^{+}$is $t=\sum_{i=1}^{n} a_{i}$.

Note that (i) means that $G$ is connected w.h.p. so $G$ has a spanning tree $T$ satisfying that $|E(T) \cap N| \leqslant t-1$. Hence if $t \leqslant\left(1 / 2-2^{-12}\right) n+o(n)$ or $t \geqslant\left(1 / 2+2^{-12}\right) n+o(n)$ then $\mathcal{D}\left(G, \mathcal{T}_{n}\right) \geqslant 2^{-12} n-o(n)$.

Three edges of $N$ are incident to each element of $A_{1}$, four edges to each of $A_{2}$. The number of edges incident to a component of size at least 3 could be less than four only if the component contains a cycle, i.e., w.h.p. only in $O(1)$ many components $A_{i}$ for $i=3, \ldots, 2^{9}$. For every component larger than $2^{9}$, and smaller than $n / 2$, w.h.p. the number of incident edges is at least four by (i).

That is, w.h.p.

$$
2|N| \geqslant 3 a_{1}+4 \sum_{i=2}^{n} a_{i}-O(1)=4 t-a_{1}-O(1),
$$

which gives

$$
\left(1 / 2-2^{-12}\right) n+O(1) \leqslant t \leqslant|N| / 2+a_{1} / 4+O(1) \leqslant 3 n / 8+a_{1} / 4+O(1) .
$$

Now we consider the number of negative edges. The number of edges in $N$ which are incident to vertices in $A_{1}$ is $e\left(G\left[A_{1}\right]\right)+e\left(G\left[A_{1}, \bar{A}_{1}\right]\right)$. Since $|N| \leqslant|P|$, we have

$$
\frac{3 a_{1}}{2} \leqslant e\left(G\left[A_{1}\right]\right)+e\left(G\left[A_{1}, \bar{A}_{1}\right]\right) \leqslant|N| \leqslant \frac{3 n}{4},
$$


which implies that $a_{1} \leqslant n / 2$. Using the condition (i), we have $e\left(G\left[A_{1}, \bar{A}_{1}\right]\right) \geqslant 2^{-7} a_{1}$. Therefore,

$$
3 a_{1} \leqslant 2 e\left(G\left[A_{1}\right]\right)+e\left(G\left[A_{1}, \bar{A}_{1}\right]\right) \leqslant 2|N|-2^{-7} a_{1},
$$

implying

$$
\frac{3 a_{1}}{2}+\frac{a_{1}}{2^{7}} \leqslant|N| \leqslant \frac{3 n}{4}
$$

which gives $a_{1} \leqslant\left(1 / 2-2^{-10}\right) n$ w.h.p. With (1) it implies $t \leqslant\left(1 / 2-2^{-12}\right) n+o(n)$ w.h.p. That gives us $\mathcal{D}\left(G, \mathcal{T}_{n}\right) \geqslant 2^{-12} n-o(n)$. w.h.p.

\section{Discrepancies of planar graphs}

Lemma 12. Let $C$ be a vertex cut of a connected graph $G$, that is $V(G)=A \cup B \cup C$ such that there are no edges between $A$ and $B$, and, say, $|A| \leqslant|B|$. Then $\mathcal{D}\left(G, \mathcal{T}_{n}\right) \leqslant$ $|B|-|A|+|C|$.

Proof. Let $f(x, y)=1$ if $(x, y) \in E(A) \cup E(A, C), f(x, y)=-1$ if $(x, y) \in E(B) \cup E(B, C)$ and arbitrary in $E(C)$. Every spanning tree $T$ of $G$ has at most $|C|$ components restricted to $A \cup C$. It means the number of edges labeled by 1 is at least $|A|+|C|-1-|C|=|A|-1$ in $T$, and the edges labeled by -1 at most $|B|+|C|-1$.

Proof of Theorem 4. To deduce Theorem 4 we need to recall the celebrated planar separation theorem of Lipton and Tarjan in [12]. It says if $G$ is a planar graph on $n$ vertices then $G$ has a vertex cut of size $O(\sqrt{n})$ partitioning the graph into two parts $A$ and $B$, where $n / 3 \leqslant|A|,|B| \leqslant 2 n / 3$. A well-known consequence [9, Theorem 5] of that theorem is that there exists a cut $C$ and constants $c_{1}, c_{2}, c_{3}$ such that $n / 2-c_{1} \sqrt{n} \leqslant|A|,|B| \leqslant n / 2+c_{2} \sqrt{n}$ and $|C|=c_{3} \sqrt{n}$.

Having the partition above we can use Lemma 12 getting that for a planar graph $G$, $\mathcal{D}\left(G, \mathcal{T}_{n}\right) \leqslant|B|-|A|+|C| \leqslant O(\sqrt{n})$.

Lemma 13 ([8]). Let $S \subseteq P_{k} \square P_{k}$ such that $\left(k^{2}-k\right) / 2 \leqslant|S| \leqslant\left(k^{2}+k\right) / 2$. Then we have $|\partial S| \geqslant k$.

Proof of Theorem 5. Assume there exists an $f: E\left(P_{k} \square P_{k}\right) \rightarrow\{-1,1\}$ such that

$$
\mathcal{D}\left(P_{k} \square P_{k}, \mathcal{T}_{n}, f\right) \leqslant k / 4
$$

Let $P, N$ and $M$ be the subset of vertices, such that $v \in P$ if all edges incident to $v$ are positive, $v \in N$ if all edges incident to $v$ are negative, and $M=V-N-P$. Consider an arbitrary Hamiltonian path in $P_{k} \square P_{k}$, from the assumption on $f$ it follows that $|P|,|N| \leqslant k^{2} / 2+k / 8+2$.

First, we show that $|M| \geqslant k$. If $\max \{|P|,|N|\} \leqslant\left(k^{2}-k\right) / 2$ then this follows from $|P|+|N|+|M|=k^{2}$. That is we may assume $\left(k^{2}-k\right) / 2<|P| \leqslant k^{2} / 2+k / 8+2$. Note that $\partial P=M$. By Lemma 13, for sets $P$ of such size we have $|\partial P| \geqslant k$, which means $|M| \geqslant k$, too. 
We identify the vertices of $P_{k} \square P_{k}$ with coordinate pairs such that $(0,0)$ belongs to the bottom left vertex, $(k-1, k-1)$ to the upper right vertex. For $r, s \in\{0,1\}$ let $X_{r, s}$ be those vertices $(i, j)(0 \leqslant i, j \leqslant k-1)$ for which $i=r(\bmod 2)$ and $j=s(\bmod 2)$. At least one of these sets $X_{r, s}$ contains at least $k / 4$ vertices of $M$, say $X_{0,0}$. Consider an arbitrary tree $T$ spanned on the vertices $X_{0,1} \cup X_{1,0} \cup X_{1,1}$.

Note that we can extend $T$ to the entire $P_{k} \square P_{k}$ such that the vertices of $X_{0,0}$ will be leaf vertices in the extension. Moreover for $(i, j) \in X_{0,0} \cap M$ we can connect $(i, j)$ to $T$ with either an edge labeled by -1 or 1 . Fixing any extension to $X_{0,0} \backslash M$, let $T^{+}$ $\left(T^{-}\right)$be the extension where we use the edge labeled by $1(-1)$ for the vertices $X_{0,0} \cap M$. Obviously, $\left|\sum_{e \in T^{+}} f(e)-\sum_{e \in T^{-}} f(e)\right| \geqslant k / 2$, so either $\left|\sum_{e \in T^{+}} f(e)\right|$ or $\left|\sum_{e \in T^{-}} f(e)\right|$ is at least $k / 4$.

Proof of Proposition 6. We show first that $\mathcal{D}\left(P_{k} \square P_{2}, \mathcal{P}\right) \geqslant k / 2$. Let us refer to the graph $P_{k} \square P_{2}$ as a rectangle with horizontal length $k$ in which the edges are labeled by $f$. Let $X$ and $Y$ be the set of the vertical edges labeled by +1 and -1 respectively. Without loss of generality, we may assume $|X| \geqslant|Y|$ and let $x:=|X| \geqslant k / 2, y:=|Y|$. We consider four paths: $P(X)$ starts from the left-upper corner goes to right except when it meets an edge $e \in X$ at which point it goes down or up, depending on which one is possible. The path $P^{\prime}(X)$ is almost the same, but it starts from the left-lower corner. Finally the paths $P(Y)$ and $P^{\prime}(Y)$ are drawn analogously, those also start from left and go to right, but rise and fall at the edges belonging to $Y$. Note that $P(X)$ and $P^{\prime}(X)$ each contain $X$, $P(Y)$ and $P^{\prime}(Y)$ each contain $Y . P(X) \cup P^{\prime}(X)$ and $P(Y) \cup P^{\prime}(Y)$ have the same set of horizontal edges.

Let $z_{1}:=\sum_{e \in P(X) \backslash X} f(e)$, and $z_{2}:=\sum_{e \in P^{\prime}(X) \backslash X} f(e)$. If $\max \left\{z_{1}, z_{2}\right\} \geqslant 0$, then we are done since one of $\sum_{e \in P(X)} f(e)$ or $\sum_{e \in P^{\prime}(X)} f(e)$ is at least $k / 2$. If both $z_{1}$ and $z_{2}$ are negative, we have $\mathcal{D}\left(P_{k} \square P_{2}, \mathcal{P}, f\right) \geqslant x+z_{1}$, and $\mathcal{D}\left(P_{k} \square P_{2}, \mathcal{P}, f\right) \geqslant x+z_{2}$. Considering the paths $P(Y)$ and $P^{\prime}(Y)$ we also have $2 \mathcal{D}\left(P_{k} \square P_{2}, \mathcal{P}, f\right) \geqslant 2 y-z_{1}-z_{2}$, since the horizontal edges in those carry exactly $z_{1}+z_{2}$ negative surplus. Adding those up, we get $4 \mathcal{D}\left(P_{k} \square P_{2}, \mathcal{P}, f\right) \geqslant 2 x+2 y$, that is $\mathcal{D}\left(P_{k} \square P_{2}, \mathcal{P}, f\right) \geqslant k / 2$ since $x+y=k$.

In the general case we may assume that $k \leqslant \ell$ and $P_{k} \square P_{\ell}$ is referred as a rectangle with $k$ rows and $\ell$ columns. We cut out $\lfloor k / 2\rfloor$ non-touching stripes $P_{2} \square P_{\ell}$. For every $f: E\left(P_{k} \square P_{\ell}\right) \rightarrow\{-1,1\}$, applying our construction of paths above, without loss of generality, at least half of the rectangles have a path with more positive edges, and with discrepancy at least $\lceil\ell / 2\rceil$. Note also, that these paths can be joined into one path by adding at most $k-1$ edges. Thus, we create a path with discrepancy at least

$$
\left\lceil\frac{1}{2}\left\lfloor\frac{k}{2}\right\rfloor\right\rceil\left\lceil\frac{\ell}{2}\right\rceil-k+1>\frac{k \ell}{8}-\frac{\ell}{8}-k
$$

and the result is proved.

Remark. Motivated by Theorem 1 and $\mathcal{D}\left(K_{n}, k \cdot K_{3}\right)=n / 5$ from [6] we think that for any $c>0, \mathcal{D}\left(G, k \cdot K_{3}\right)=\Theta(n)$ provided that $v(G)=n$ and $\delta(G) \geqslant(3 / 4+c) n$. 


\section{References}

[1] J. R. Alexander, J. Beck, and W. W. L. Chen. Geometric discrepancy theory and uniform distribution. In Handbook of Discrete and Computational Geometry, 1997.

[2] J. Beck. On size Ramsey number of paths, trees, and circuits. I. Journal of Graph Theory, 7(1):115-129, 1983. https://doi.org/10.1002/jgt.3190070115.

[3] J. Beck and W. W. L. Chen. Irregularities of Distribution. Vol. 89 of Cambridge Tracts in Mathematics, Cambridge University Press, 1987.

https://doi .org/10.1017/CB09780511565984.

[4] B. Bollobás. A probabilistic proof of an asymptotic formula for the number of labelled regular graphs. European Journal of Combinatorics, 1:311-316, 1980. https://doi .org/10.1016/S0195-6698(80)80030-8.

[5] B. Bollobás. The isoperimetric number of random regular graphs. European Journal of combinatorics, 9(3):241-244, 1988.

https://doi .org/10.1016/S0195-6698(88)80014-3.

[6] S. A. Burr, P. Erdős and J. H. Spencer. Ramsey theorems for multiple copies of graphs. Transactions of the American Mathematical Society, 209:87-99, 1975. https://doi.org/10.2307/1997371.

[7] P. Buser. On the bipartition of graphs. Discrete Applied Math., 9:105-109, 1984. https://doi .org/10.1016/0166-218X (84)90093-3.

[8] J. Chvátalová. Optimal labelling of a product of two paths. Discrete Mathematics, 11(3):249-253, 1975. https://doi .org/10.1016/0012-365X (75)90039-4.

[9] H. N. Djidjev. On the problem of partitioning planar graphs. SIAM Journal of Algebraic and Discrete Methods, 3:229-241, 1982. https://doi.org/10.1137/0603022.

[10] P. Erdős, M. Goldberg, J. Pach and J. Spencer. Cutting a graph into two dissimilar halves. Journal of Graph Theory, 12:121-131, 1988.

https://doi.org/10.1002/jgt.3190120113.

[11] P. Erdős, Z. Füredi, M. Loebl and V. T. Sós. Discrepancy of Trees. Stud. Sci. Math., 30:47-57, 1995.

[12] R. J. Lipton and R. E. Tarjan. A separator theorem for planar graphs. SIAM Journal on Applied Mathematics, 36:177-189, 1976. https://doi.org/10.1137/0136016.

[13] L. Pósa. On the circuits of finite graphs. Magyar. Tud. Akad. Mat. Kutató Int. Közl., 8:355-361, 1963/1964.

[14] H. Weyl. Über die Gleichverteilung von Zahlen mod Eins. Math. Ann., 77:313-352, 1916. https://doi.org/10.1007/BF01475864.

[15] N. C. Wormald. Models of random regular graphs. London Mathematical Society Lecture Note Series, 239-298, 1999. 